\title{
How to design a Randomised Controlled Trial
}

Brocklehurst, Paul; Hoare, Zoe

\section{British Dental Journal}

DOI:

10.1038/sj.bdj.2017.411

Published: 01/05/2017

Peer reviewed version

Cyswllt i'r cyhoeddiad / Link to publication

Dyfyniad o'r fersiwn a gyhoeddwyd / Citation for published version (APA):

Brocklehurst, P., \& Hoare, Z. (2017). How to design a Randomised Controlled Trial. British Dental Journal, 222, 721-726. https://doi.org/10.1038/sj.bdj.2017.411

\footnotetext{
Hawliau Cyffredinol / General rights

Copyright and moral rights for the publications made accessible in the public portal are retained by the authors and/or other copyright owners and it is a condition of accessing publications that users recognise and abide by the legal requirements associated with these rights.

- Users may download and print one copy of any publication from the public portal for the purpose of private study or research.

- You may not further distribute the material or use it for any profit-making activity or commercial gain

- You may freely distribute the URL identifying the publication in the public portal ?
}

Take down policy

If you believe that this document breaches copyright please contact us providing details, and we will remove access to the work immediately and investigate your claim. 


\section{How to design a Randomised Controlled Trial?}

Professor Paul Brocklehurst BDS, FFGDP, MDPH, PhD, FDS RCS

Director of NWORTH Clinical Trials Unit and Honorary Consultant in Dental Public Health

Dr Zoe Hoare BSc, PhD

Principal Statistician, NWORTH Clinical Trials Unit

Contact:

Professor Paul Brocklehurst

Director of NWORTH Clinical Trials Unit

http://nworth-ctu.bangor.ac.uk

@NWORTH CTU 


\section{Introduction}

Randomised controlled trials (RCTs) are the work-horse of evidence-based healthcare and the only research design that can demonstrate causality i.e. that an intervention causes a direct change in a clinical outcome. Although they can be complex, the idea at its simplest is to "create two identical systems into one of which a new component [the intervention] is introduced". ${ }^{1}$ Observations are then made of outcome differences that occur between experimental and control conditions and "should a change occur, it is attributed to the one difference between them" (Figure 1). ${ }^{1}$ This paper aims to explain how to design a Randomised Controlled Trial for those who have little prior knowledge of the topic and specifically explore the following areas: will focus on some of the important issues of trial design and will cover:

- The PICO statement

- Randomisation

- Trial design

- Statistical testing

- Sample size calculations

- Bias

- Clinical Trials Units

\section{Before you start: how do you ask the right question?}

Whether you are designing an individually randomised, cluster randomised, stepped wedge or adaptive trial, your research question always returns to your PICO statement. Precision in defining a research question is a key skill; the more precise the research question is, the easier it is to design your study. The PICO statement divides a research question into four basic parts: the patient/population (who are you intending to conduct the study with/on), the intervention, the control and the outcome measure.

\section{$P=$ Population}

The first step in developing a well-built question is to identify the patient problem or describe the population group of interest. When identifying the P in PICO it is helpful to ask yourself how you would describe this population group to another person. What are their important characteristics of the group? For example, this could be 
children, or more specifically young children under the age of five years of age. For example, in the Northern Ireland Caries Prevention In Practice (NIC-PIP) trial, the eligible population was caries free children aged between two and three years of age (Table 1). ${ }^{2}$

\section{$I=$ Intervention}

Identifying the intervention is the second step in the PICO process. It is important to identify what you plan to do to your population. This may include the use of a specific diagnostic test, treatment, adjunctive therapy, medication or a recommendation to use a product or procedure. When thinking about conducting a randomised controlled trial, the intervention would be the health technology that you intend to test experimentally. In NIC-PIP, this was the delivery of a preventive regime in line with Delivering Better Oral Health. In the iQuaD trial, the intervention was personalised oral hygiene advice (Table 1 ). ${ }^{3}$ Some RCTs also employ more that one active arm simultaneously. For example, the FiCTION trial utilises two active arms: conventional caries management with best practice prevention and biological management of caries with best practice prevention (Table 1). ${ }^{4}$

\section{$I=$ Control or comparison}

The control or comparison is the third step to take in building your PICO question. This represents the alternative you are planning to compare the intervention to. This can take a number of forms. For example, it could be no active intervention. This is classically known as the "control group" in a RCT. For example, you might be comparing a new high fluoride toothpaste to prevent dental caries. However, it could equally refer to present current treatment. Here, the researcher would be testing a new intervention drug compared to existing treatment an old drug or two different types of intervention like Atraumatic Restorative Treatment versus the Hall Technique, in what is called a "head-to-head". ${ }^{5}$

\section{O = Outcome}

Determining the primary outcome measure (POM) is the final step in building the PICO question and one of the most important as it has ramifications on how you statistically test for differences between the intervention and the control/comparator. It specifies what you would expect to see, should the intervention be successful. It is important to decide here whether your POM would be measured using this will be a continuous variable or an ordinal one. The difference between these two types of 
variables is that a continuous variable describes outcomes that are measured on a scale, like height or weight, whereas ordinal variables are categorical in nature and as the name suggests, can be placed in order. For example, if a person is asked about their feelings towards their dental care and the available responses are unsatisfied, neutral or satisfied, this would be an ordinal variable.

Another key aspect to specify when thinking about your POM is its time to expression i.e. how quickly and when you would expect to see your result (which will be dependent on the "time-to-expression" of the intervention in your population). Time to expression has a critical influence on the duration of the trial (and thereby cost) and will obviously vary with the type of disease under investigation. For example, trials evaluating interventions for gingivitis will have a much shorter duration compared to caries trials. In the FiCTION trial, the research question is "what is the clinical and cost effectiveness of restoration caries in primary teeth, compared to no treatment?". Here, the POM is the incidence of either pain or infection related to dental caries and the follow-up period is three years (Table 1).

\section{Why bother with randomisation?}

When a new RCT is being planned, researchers are said to be in equipoise. This means that we are uncertain whether the new treatment being experimentally tested actually produces a benefit for the participant. This is an ethical position. If we already have evidence that a new treatment is better than another, we should be giving this treatment to the patient already and if we know there is no difference or that the new treatment is harmful, we shouldn't be offering it all to the patient. Consolidated Standards of Reporting Trials (CONSORT) 2010 states "ideally, participants should be assigned to comparison groups in the trial on the basis of a chance (random) process characterized by unpredictability." ${ }^{6}$ The requirement is there for a reason. Randomisation of the participants is crucial because it allows the principles of statistical theory to stand and as such allows a thorough analysis of the trial data without bias.

So, how do we randomise? Surely putting participants into random groups is as simple as tossing a coin? This is randomisation in its simplest form but in many cases it results in an unbalanced sample. For example, in a small trial of say 50 participants, tossing a fair coin 50 times would result in a $25: 25$ split only $7.95 \%$ of the time! 
There are many different types of randomisation. Tossing a coin or using a random number table are examples of simple randomisation. Restricted randomisation uses methods to control the imbalance between the groups; generating a random list AAABBBABABAABB allows participants to be allocated as they arrive to the next treatment on the list. With the list here we know that at the sixth, eighth, tenth and 14th participants we have balance in allocation. Stratified randomisation allows us to account for and control certain characteristics within the population of participants such as gender or age (factors that might confound the final effect). It is now recommended that stratification should be used sparingly and only in those characteristics that you think would potentially affect your outcome. With four stratification variables each with 2 levels, you would be dividing your sample into $4^{z}$ sub-samples and the question is then: how likely is it that each of those 16 combinations would be fairly represented? Given that you never know which patient will walk through the door next, you could end up with a completely unbalanced design anyway.

When choosing a randomisation method it is important to determine whether the method can accommodate enough treatment groups. For example, tossing a coin would be difficult to implement for a trial with three arms three group trial. It is also important to determine how predictable the method is. A deterministic algorithm (not considered randomisation) would allow you to be able to predict what treatment would be allocated next. A static random element would mean that each allocation is made with a pre-determined probability (tossing a coin gives a 50:50 chance of either treatment being assigned). A dynamic element adjusts the probability of being allocated to a treatment based on what has already been allocated in the trial so far. This is the basis of the North Wales Organisation for Randomised Trials in Health's (NWORTH) remote randomisation system. ${ }^{7}$

Other considerations include, can the method accommodate for stratification variables and if so, how many? Can the method handle an unequal allocation ratio? Is thresholding used (i.e. maximum level of accepted imbalance)? Can the method be implemented sequentially i.e. as the patients walk through your door? Is the method complex? Is the method suitable for cluster randomisation? Decisions like these mean that often a Clinical Trials Unit is needed in the design and planning of your trial. Further reading can be found here. ${ }^{8,9}$ 


\section{How do you go about designing your trial?}

There are different phases of RCTs. Phase I trials are described as "first into person", whilst Phase II are slightly larger trials that commonly determine efficacy i.e. does the intervention work or not. Phase III trials take this a step further and determine effectiveness i.e. does the intervention produce health benefits in the real world. This section will focus on Phase III trial designs. Again, it is important that you consult a statistician at this stage: "to call in the statistician after the experiment is done may be no more than asking him to perform a post-mortem examination: he may be able to say what the experiment died of". ${ }^{10}$

\section{Feasibility or pilot or full trial}

A key question to ask when designing a trial is do you have all the information to inform all the parameters needed. A feasibility study helps you determine whether a definitive trial is "feasible". This type of study is often not randomised. This is because the intervention under study is commonly under development and the study plan or intervention itself would change before a definitive study is started. The important outcomes of a feasibility study will be things like the ability to recruit participants, ability to retain participants for the length of time required, the suitability of the proposed outcome measures, and willingness of the participants /clinicians to be involved.

Pilot studies can be thought of as a small versions of the definitive trial i.e. your intervention is now established, but there is still some uncertainty about whether your definitive trial can run as planned. A pilot study will assess all the features that a feasibility study does and often the terms are used interchangeably. Further reading on the issue of terminology can be found here. ${ }^{11,12,13}$ With a stable intervention to test, enough information about the difference in the primary outcome measure-POM you expect (known as the effect size), enough understanding about the time to expression of your chosen POM, confidence about the feasibility of running the trial as designed, then it may be time to design a definitive trial of the intended intervention.

\section{Randomised or non-randomised}

A RCT randomised controlled trial is seen as the 'gold standard' of trial design but there are some situations where randomisation is not possible for logistical or ethical reasons and so it can be acceptable to design a non-randomised trial. Uncontrolled 
or non-randomised trials are used when randomisation is not possible or is unethical. The results of non-randomised or uncontrolled trials may be considered less reliable as there is an increased risk for errors affecting the outcome of the trial. An example of a non-randomised trial is Lam et al's (2010) study of mental-health first aid training. ${ }^{14}$ The "SPIRIT 2013 Statement" provides recommendations for a minimum set of scientific, ethical, and administrative elements that should be addressed in a clinical trial protocol. ${ }^{15}$ It is worth remembering that a non-randomised the trial will have to be analysed and reported very differently if randomisation is not a key component. All trials should be reported to CONSORT standards (CONSORT stands for Consolidated Standards of Reporting Trials) and it worth keeping these guidelines for reporting in mind during the design process. ${ }^{10}$

A key question with RCTs is whether the randomisation is at an individual level or at a cluster level. The individually randomised parallel group design is typically seen as the standard RCT design and remains the favoured approach by funders. An example of an individually randomised parallel group design in dentistry is the FiCTION trial. ${ }^{5}$ This is appropriate IF the intervention is to be delivered to an individual and there is no possibility of contamination. However, this is not always possible. For example, if a community based oral health prevention programme was being delivered in a school, it would be difficult to undertake the intervention on one child and not affect another child. The environment in the school and the teachers that undertook the intervention would find it difficult not to influence a child in the control group. In such cases, cluster randomisation would be used. In this example, schools would be the unit of randomisation, not the individual (so a whole school would initiate the intervention and a whole school wouldn't). Two examples of cluster randomised trials in dentistry are NIC-PIP and IQuaD. ${ }^{2,3}$

Another design of definitive trial is a stepped wedge trial. Here, the unit of randomisation is not isn't the individual or the cluster, but time. This might sound complex, but all this means is that everyone gets the intervention, but just not yet. These designs are not aren't commonly used, but have the advantage that they map on well to a policy roll-out, where everyone will get the intervention. As a result, there are ethical advantages to the approach. For example, if a new practice prevention tool from Public Health England was going to be introduced across England and could be rolled out on a staggered basis, this approach could be undertaken. At specific time-points in the trial (the "step"), participating practices that are not yet 
adopting the intervention (who are currently acting as the control) come "on-line". The down-side of stepped-wedge trials is that as each new wave of practices adopt the intervention, all the recruited practices in the trial have to have the POM measured again. Another disadvantage of this design is that you must have sufficient time between each step for the disease of interest to express itself. If this was a gingivitis measure, then this would not be too problematic, but if the researchers were examining the impact of the preventive intervention on dental caries in adults, this would mean that the trial would be very long. One example of a dental stepped wedge trial is the SOCLE-II trial. ${ }^{16}$ Here, researchers are exploring whether enhanced oral healthcare or usual oral healthcare is the most effective for people in stroke care settings. Rather than randomising the participants into the two groups, they are rolling out the intervention (enhanced oral healthcare), one ward at a time. More information on stepped-wedge trials can be found here. ${ }^{17,18}$

\section{How do you go about demonstrating your intervention has worked?}

A statistical hypothesis in a trial describes what the researcher expects to see happen to their chosen POM as their intervention is applied to the intervention arm. This assumption may or may not be true. The null hypothesis assumes that changes in the POM result from chance and that there is no difference between the intervention and the control arm. The alternate hypothesis assumes that changes are influenced by some non-random cause i.e. the intervention the researcher has introduced has worked!

Individually, cluster and stepped-wedged designs commonly test a directional hypothesis that the new intervention produces a health benefit compared to the control or an existing intervention ("head-to-head"). These are termed "superiority designs" and from a statistical perspective, test whether your point estimate (mean if the POM is measured using a continuous variable) lies above or below the $95 \%$ confidence interval $(\mathrm{Cl})$ in the control arm. However, sometimes our question is whether a new treatment is as good as another treatment or meets a certain standard. Trials that explore these issues are known as "equivalence" or "noninferiority" designs. "Equivalence" trials determine whether the value for the POM in both arms is not statistically different i.e. that the $95 \% \mathrm{Cl}$ of the difference of the two groups lies within an acceptable margin (the equivalence margin). "Non-inferiority" designs test the difference between two arms and test whether the new intervention is not unacceptably worse than the other i.e. that the lower end $95 \% \mathrm{Cl}$ of the 
difference in POM does not extend below the pre-defined non-inferiority margin. More reading can be found here. ${ }^{19}$

Statistical tests commonly quote the "p" value, which describes the statistical significance of the results. The $p$ value is the probability that the difference between the scores would have happened by chance, therefore the lower the $p$ value, the more likely that there is a significant difference between the scores. It is generally accepted that any POM being tested is statistically significant if the $p$ value is below 0.05. If this is the case, the null hypothesis can be rejected. However, it is worth knowing that the more times you test something, the more likely by chance you are going to find something statistically significant. In the cases of multiple testing, consideration should be given to adjusting the level of significance.

\section{How many participants do you need in your trial?}

There are three elements to a sample size calculation: the p-value, power and the effect size. The p-value is set commonly at 0.05 (as highlighted above). Power is the probability that you will see an effect IF an effect is there to be seen. Sometimes we don't see a statistically significant effect because quite simply, no effect exists. However, sometimes an effect is there, but we don't have the numbers to see it (this is called being under-powered). For RCTs, we set this probability of detecting an effect IF an effect is there to be seen at $90 \%$. The only element that varies in the power calculation is the effect size i.e. this is the main element that the researcher needs to determine in consultation with a statistician. An effect size is a point estimate of the measure of the strength of effect standardised by the variability of the measure i.e. the expected difference in your POM between the intervention and control arm (for example, if your POM is measured using a continuous variable, this would commonly be the mean with the variability represented by the standard deviation).

Table 1 and Table 2 provides you with the details that a statistician would need to know before a sample size can be calculated. More reading can be found here. ${ }^{20,21}$

\section{What about bias?}

Common to all trial designs is the need to reduce bias. A bias is a systematic error and can operate in either direction: under or over-estimating the true intervention effect. Bias is caused by flaws in the design of the study and so is not the same as 
imprecision, which is a random error. Selection bias refers to systematic differences between the intervention and control arm caused by differences in baseline characteristics. This should be removed if randomisation process was effective. Ensuring that participants are blind to their allocation (where possible) reduces the risk that knowledge of which intervention was received, rather than the intervention itself, affects the outcome. This is called allocation concealment. Detection bias (or ascertainment bias) refers to systematic differences produced by differences in how outcomes are determined. Blinding of outcome assessors may reduce the risk that knowledge of the intervention, rather than the intervention itself, affects the POM. Blinding of outcome assessors is especially important when subjective POMs are used, for example, how nervous were you during your dental treatment?

Attrition bias describes the systematic differences between the intervention and control arm caused by withdrawals from the trial i.e. when the participants do not want to take part anymore. This can skew the numbers and mix of participants in each arm. It may also tell you that your trial is not socially acceptable! Reporting bias (or publication bias) refers to systematic differences caused by researchers and journals only reporting positive effects of intervention. ${ }^{22}$ This can be seen in the pharmaceutical industry where negative results about the effects of a particular drug can get hidden. ${ }^{23}$

\section{What are Clinical Trials Units}

Clinical Trials Units (CTUs) are "specialist units which have been set up with a specific remit to design, conduct, analyse and publish clinical trials and other welldesigned studies" (https://youtu.be/QvGaGEHgwXg). ${ }^{24}$ Commonly, they have a number of functional areas:

- $\quad$ Statistical support (pre, per and post-trial)

- Trial Management

- Quality Assurance

- Information Technology

CTUs have expertise in the co-ordination of trials, particularly those that involve Investigational Medicinal Products, where compliance with the Medicines Health Regulatory Authority is critical to discharge the expectations in the "UK Medicines for 
Human Use (Clinical Trials) Regulations". ${ }^{25}$ Some also provide specialist expert statistical for clinicians. For example, although NWORTH has over $£ 18 \mathrm{M}$ of trials on its portfolio from across the United Kingdom, it is also part-funded by the Welsh Government to provide the Research Design and Conduct Service (http://nworthctu.bangor.ac.uk/research-support-service/index.php.en).

Most CTUs, but not all, are registered with the United Kingdom Clinical Research Collaboration (UKCRC) and many specialise in specific areas, like Clinical Trials of Investigative Medicinal Products (drug trials) or cancer trials. NWORTH has a traditional strength in pragmatic trials and trials of complex interventions.

Methodologically, they link with initiatives like TrialForge (http://www.trialforge.org) and work to understand how to "make trials work" (see http://nworthctu.bangor.ac.uk/trials.php).

When preparing for a grant application, researchers are encouraged to approach CTUs early to get help on designing their project. The National Institute of Health Research sees CTUs "as an important component of any research application and funded project" and you are expected to inform them whether you have contacted a CTU in any grant application. They also provide a useful schematic of the necessary steps to take when planning a definitive trial (http://www.ct-toolkit.ac.uk).

\section{In summary}

This paper has explored the key design elements of RCTs. Although there are significant challenges encountered when designing such complex studies, thinking through each component described above will provide clarity and hopefully encourage more GDPs to get involved in research. ${ }^{26}$ This is important as there is an increasing need for high-quality evidence from primary care settings to guide the delivery of future healthcare. 
Table 1: Examples of PICO statements for recent/ongoing trials conducted in a primary care environment

\begin{tabular}{|c|c|c|c|c|c|}
\hline Trial & Population & Intervention & Control/Comparator & $\begin{array}{c}\text { Clinical primary outcome } \\
\text { measure }\end{array}$ & Follow-up period \\
\hline iQuaD & $\begin{array}{l}\text { Regularly attending NHS } \\
\text { dentate adult patients who have } \\
\text { periodontal health, gingivitis or } \\
\text { moderate periodontitis }\end{array}$ & $\begin{array}{l}\text { Personalized oral hygiene } \\
\text { advice }\end{array}$ & Standard oral hygiene advice & $\begin{array}{l}\text { Gingival inflammation/bleeding } \\
\text { on probing at the gingival margin } \\
\text { AND oral hygiene self-efficacy }\end{array}$ & Three years \\
\hline NIC-PIP & $\begin{array}{l}\text { Children aged } 2-3 \text { years, who } \\
\text { were caries free at baseline }\end{array}$ & $\begin{array}{l}\text { Fluoride varnish, a toothbrush } \\
\text { and a 50-ml tube of adult } \\
\text { toothpaste and standardized } \\
\text { evidence-based prevention } \\
\text { advice provided at 6-monthly } \\
\text { intervals }\end{array}$ & Prevention advice alone & $\begin{array}{c}\text { Proportion of children who } \\
\text { converted from caries-free to } \\
\text { caries-active states }\end{array}$ & Three years \\
\hline INTERVAL & $\begin{array}{l}\text { Regularly attending NHS } \\
\text { dentate adult patients }\end{array}$ & $\begin{array}{c}\text { Risk based recall interval based } \\
\text { on the patient's risk of dental } \\
\text { disease AND } \\
24 \text { month recall interval }\end{array}$ & 6 month recall interval & $\begin{array}{c}\text { Gingival bleeding AND quality of } \\
\text { life (OHIP-14) }\end{array}$ & Four years \\
\hline FiCTION & Children aged $3-7$ years & $\begin{array}{l}\text { Conventional caries } \\
\text { management with best practice } \\
\text { prevention AND biological } \\
\text { management of caries with best } \\
\text { practice prevention }\end{array}$ & Best practice prevention alone & $\begin{array}{l}\text { Incidence of either pain or } \\
\text { infection related to dental caries }\end{array}$ & Three years \\
\hline
\end{tabular}


Table 2: Statistical considerations when determining a sample size

\begin{tabular}{|l|l|}
\hline$\#$ & Question \\
\hline $\mathbf{1}$ & $\begin{array}{l}\text { How large do you expect the difference to be between the intervention and } \\
\text { control group to be? Is this clinically significant i.e. a difference of } 1 \mathrm{~mm} \text { in } \\
\text { probing depth may be statistically significant, but is it really important in } \\
\text { practice? If the effect size is small, the sample size will be large and vice versa. }\end{array}$ \\
\hline $\mathbf{2}$ & $\begin{array}{l}\text { How much variation do you expect to see in the difference in the POM between } \\
\text { the intervention and control arm? If the variability is small then the sample size } \\
\text { will be small, whereas if the variability is large then the sample size will be } \\
\text { large. }\end{array}$ \\
\hline $\mathbf{3}$ & $\begin{array}{l}\text { What direction will the effect be? For example, do you think your intervention } \\
\text { will produce a positive or negative difference in your POM, compared to the } \\
\text { control (or will it be indifferent). }\end{array}$ \\
\hline $\mathbf{4}$ & $\begin{array}{l}\text { Are you going to record the POM at baseline and at the end of your trial in the } \\
\text { same participants (known as "repeated measures") or in a different sample. The } \\
\text { former is common with clinical interventions, whilst the latter is more common } \\
\text { with public health interventions at a population level, where it is impractical to } \\
\text { measure the same group of people at the end of the trial compared to at } \\
\text { baseline. The latter requires a larger sample size. }\end{array}$ \\
\hline $\mathbf{5}$ & $\begin{array}{l}\text { Are the same number of participants required in both the intervention and } \\
\text { control arm, or are more participants are required in one group compared to the } \\
\text { other. Unequal allocation to groups will require a larger sample size than equal }\end{array}$ \\
\hline
\end{tabular}


Table 3: Non-statistical considerations when determining a sample size

\begin{tabular}{|l|l|}
\hline$\#$ & Question \\
\hline $\mathbf{1}$ & $\begin{array}{l}\text { Can you recruit the number of participants required from the sample size } \\
\text { calculation. This can be problematic, particularly with rare disorders. }\end{array}$ \\
\hline $\mathbf{2}$ & $\begin{array}{l}\text { Is it "do-able" in terms of costs. The cost of the study needs to be considered } \\
\text { and whether there will be sufficient funds to conduct a study with the number of } \\
\text { participants required. }\end{array}$ \\
\hline $\mathbf{3}$ & $\begin{array}{l}\text { How long does your study need to run for? This is linked to the "time to } \\
\text { expression" of your POM. It is also influenced by how quickly you can recruit } \\
\text { the numbers of participants that meet the eligibility criteria of your trial. }\end{array}$ \\
\hline $\mathbf{4}$ & $\begin{array}{l}\text { Do you need to run a pilot/feasibility study first? Do you have enough } \\
\text { information to reliably calculate a sample size? }\end{array}$ \\
\hline $\mathbf{5}$ & $\begin{array}{l}\text { How many participants might withdraw from the study before the study is } \\
\text { completed (attrition of the study). This depends on the study and the population } \\
\text { of participants. As an example, if the study is in an elderly population then you } \\
\text { would need to consider that participants may pass away before the study is } \\
\text { completed. Remember there is a difference between withdrawing from a } \\
\text { 'treatment' and withdrawing from the study and data should be collected on all } \\
\text { participants as long as possible regardless of their level of participation. }\end{array}$ \\
\hline
\end{tabular}




\section{FIGURES}

Figure 1: The experimental approach to evaluation

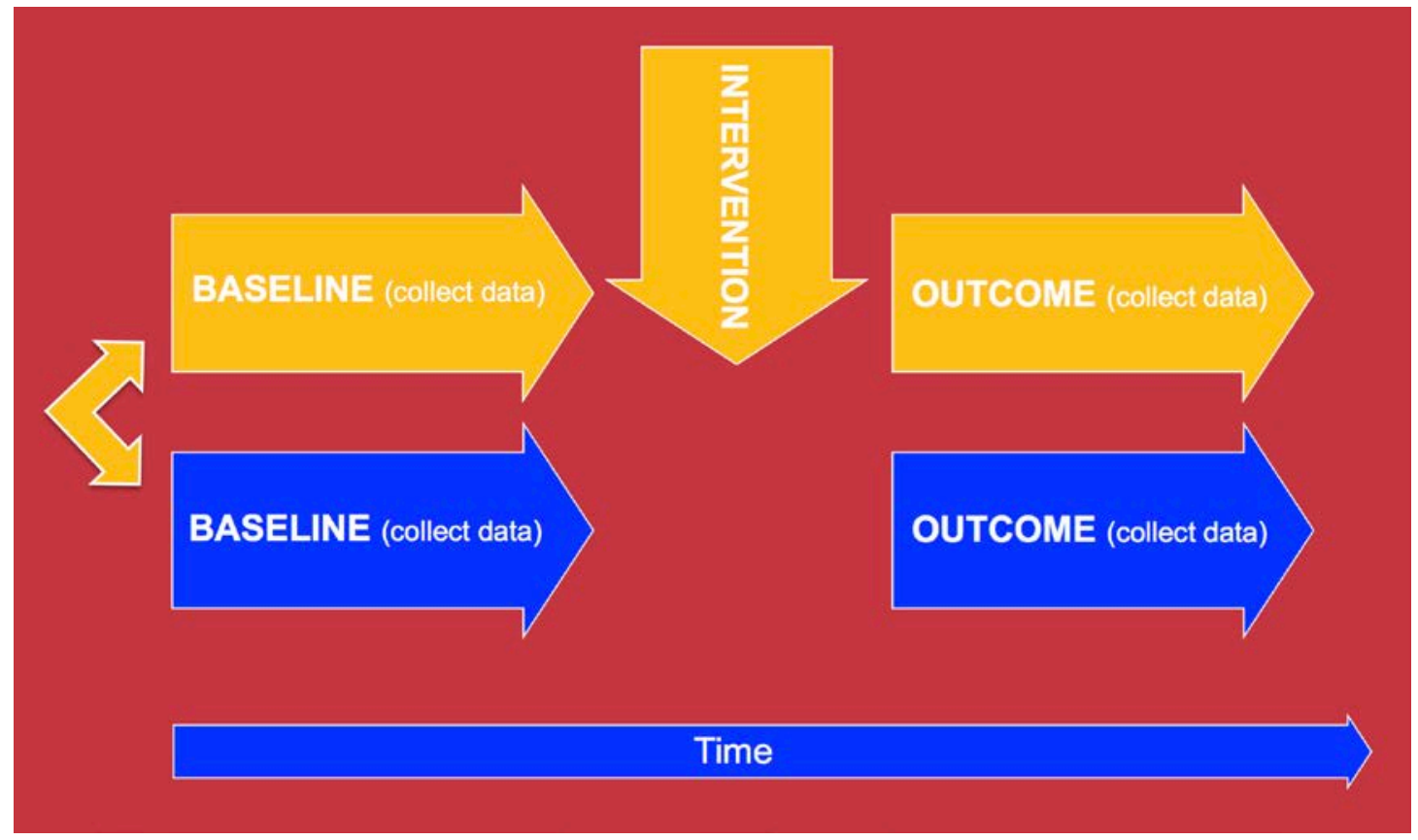

Figure 2: Trials in context

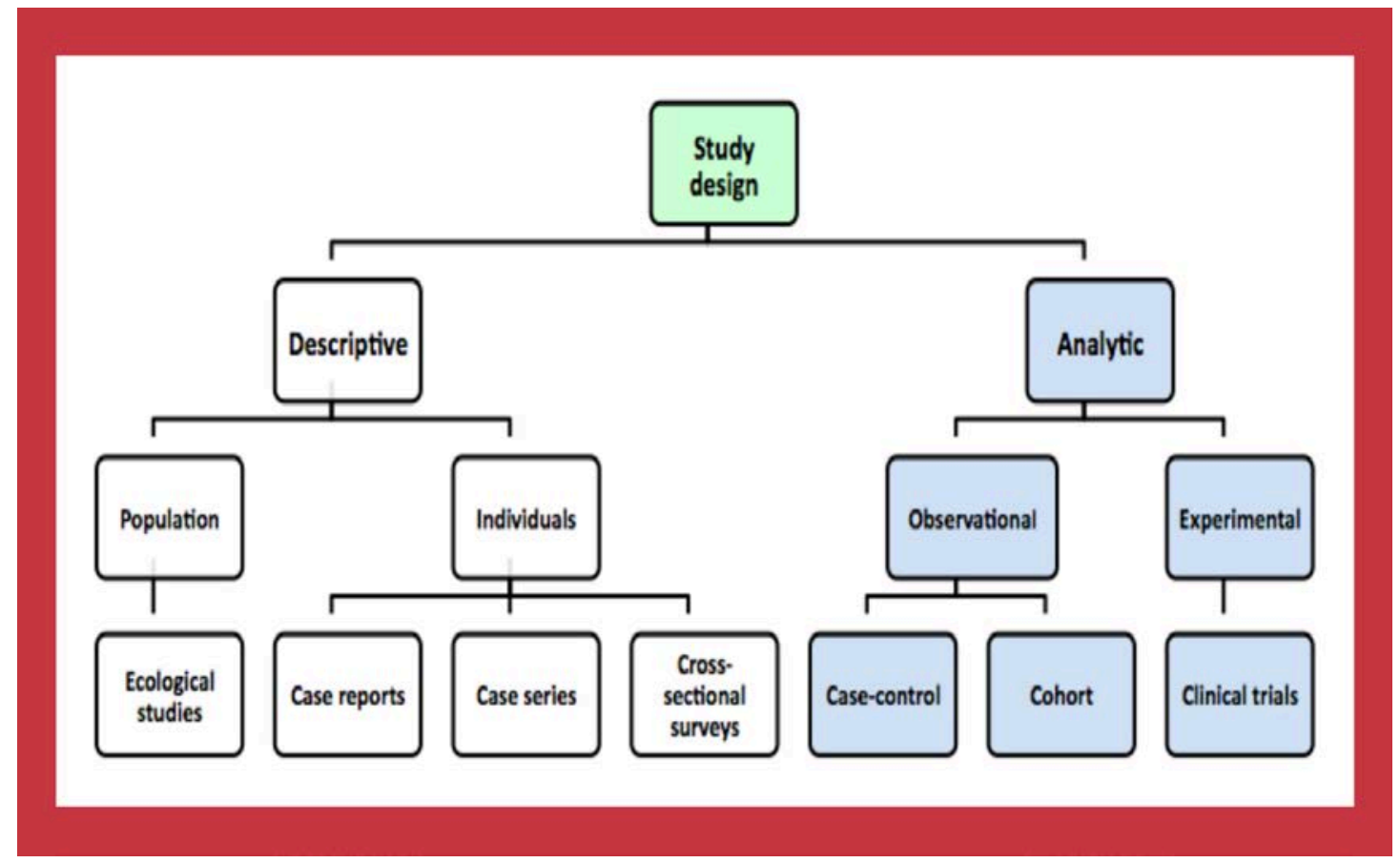




\section{REFERENCES}

${ }^{1}$ Pawson R. The Science of Evaluation. London: Sage Publications; 2013.

2 Tickle M, O'Neill C, Donaldson M, Birch S, Noble S, Killough S, et al. A randomised controlled trial to measure the effects and costs of a dental caries prevention regime for young children attending primary care dental services: the Northern Ireland Caries Prevention In Practice (NIC-PIP) trial. Health Technol Assess 2016;20(71).

${ }^{3}$ Clarkson JE, Ramsay CR, Averley P, Bonetti D, Boyers D, Campbell L, et al. IQuaD dental trial; improving the quality of dentistry: a multicentre randomised controlled trial comparing oral hygiene advice and periodontal instrumentation for the prevention and management of periodontal disease in dentate adults attending dental primary care. BMC oral health. 2013;13(1):58.

${ }^{4}$ Innes N P T, Clarkson J E, Speed C, Douglas G V, Maguire A, FiCTION Trial Collaboration. The FiCTION dental trial protocol filling children's teeth: indicated or not? BMC Oral Health 2013;13: 25.

${ }^{5}$ Hesse, D, de Araujo, MP, Olegário, IC, Innes, N, Raggio, DP \& Bonifácio, CC 2016, 'Atraumatic Restorative Treatment compared to the Hall Technique for occlusoproximal cavities in primary molars: study protocol for a randomized controlled trial' Trials, vol 17, 169. DOI: 10.1186/s13063-016-1270-z

${ }^{6}$ CONSORT 2010 Statement: updated guidelines for reporting parallel group randomised trials. Schulz KF, Altman DG, Moher D, for the CONSORT Group BMJ 2010;340:c332.

7 NWORTH remote randomisation system. Available at: http://nworthctu.bangor.ac.uk/randomisation/index.php.en. Downloaded 26 January 2017. ${ }^{8}$ Hoare Z, Whitaker CJ \& Whitaker R. Introduction to a generalized method for adaptive randomization in trials. Trials 2013;14:19. DOI: 10.1186/1745-6215-14-19. ${ }^{9}$ Russell D, Hoare ZSJ, Whitaker R, Whitaker CJ, Russell IT. Generalized method for adaptive randomization in clinical trials. Statistics in Medicine 2011;30(9):922-934. ${ }^{10}$ Fisher RA. Presidential Address by Professor RA Fisher. Sankhyā. Ind J Statistics 1938;4(1):14-17.

${ }^{11}$ Arain M, Campbell MJ, Cooper CL, Lancaster GA. What is a pilot or feasibility study? A review of current practice and editorial policy. BMC Medical Research Methodology 2010;10:67. 1471-2288/10/67

12 Lancaster GA. Pilot and feasibility studies come of age! Pilot and Feasibility Studies 2015;1:1. 
${ }^{13}$ Eldridge SM, Lancaster GA, Campbell MJ et al. Defining Feasibility and Pilot Studies in Preparation for Randomised Controlled Trials: Development of a Conceptual Framework. PLoS ONE 2016;11(3): e0150205.

doi:10.1371/journal.pone.0150205

${ }^{14}$ Lam AY, Jorm AF, Wong DF. Mental health first aid training for the Chinese community in Melbourne, Australia: effects on knowledge about and attitudes toward people with mental illness. Int J Mental Health Systems 2010;4:18.

${ }^{15}$ SPIRIT 2013 Statement: Defining standard protocol items for clinical trials. Chan A-W et al Ann Intern Med 2013;158:200-207.

${ }^{16}$ Brady MC, Stott D, Weir CJ, Chalmers C, Sweeney P, Donaldson C, Barr J, Barr M, Pollock A, McGowan S, Bowers N, Langhorne P. Clinical and cost effectiveness of enhanced oral healthcare in stroke care settings (SOCLE II): a pilot, stepped wedge, cluster randomized, controlled trial protocol. Int J Stroke. 2015 Aug;10(6):979-84. doi: 10.1111/ijs.12530. Epub 2015 Jun 16.

${ }^{17}$ Brown CA, Lilford RJ. The stepped wedge trial design: a systematic review. BMC Medical Research Methodology 2006;6:54.

${ }^{18}$ Woods B, Russell I. Randomisation and chance-based designs in social care research. Available at: http://sscr.nihr.ac.uk/PDF/MR/MR17.pdf. Downloaded 26 January 2017.

${ }^{19}$ Schumi J, Wittes JT. Through the looking glass: understanding non-inferiority. Trials. 2011 May 3;12:106. doi: 10.1186/1745-6215-12-106.

${ }^{20}$ Ellis, P. D. (2010). The essential guide to effect sizes: Statistical power, metaanalysis, and the interpretation of research results. Cambridge University Press. ${ }^{21}$ Ellis, P. D. (2010). The essential guide to effect sizes: Statistical power, metaanalysis, and the interpretation of research results. Cambridge University Press. ${ }^{22}$ Chan AW, Altman DG. Identifying outcome reporting bias in randomised trials on PubMed: review of publications and survey of authors. BMJ 2005; 330: 753.

${ }^{23}$ Goldacre B. Bad Science. Harper Collins: London; 2008.

${ }^{24}$ Clinical Trials Units. UKCRC. Available at: http://www.ukcrcctu.org.uk/?page=CTURole . Downloaded 26 January 2017. ${ }^{25}$ UK Medicines for Human Use (Clinical Trials) Regulations. Available at: http://www.legislation.gov.uk/uksi/2004/1031/contents/made. Downloaded 26 January 2017. 
${ }^{26}$ Martin-Kerry JM, Lamont TJ, Keightley A, Calache H, Martin R, Floate R, Princi K, de Silva AM. Practical considerations for conducting dental clinical trials in primary care. Brit Dent J 2015;218(11):629-634. 